\title{
FOURIER INVERSION FOR PIECEWISE SMOOTH FUNCTIONS IN SEVERAL VARIABLES
}

MARK A. PINSKY

(Communicated by George C. Papanicolaou)

\section{INTRODUCTION AND STATEMENT OF RESULTS}

Recently we have become aware [GP, PST] of the pointwise divergence of the Fourier and Fourier-Bessel series of some piecewise constant functions in three dimensions and higher. In this note we formulate these ideas in terms of the Fourier transform. The main result gives precise necessary and sufficient conditions for the pointwise convergence of the spherical partial sums of the Fourier transform, as follows.

Theorem. Let $f: \mathbf{R}^{n} \rightarrow \mathbf{R}$ be a real-valued function that is zero for $\{\mathbf{x}:|\mathbf{x}|>a\}$ and is defined by a $C^{k+2}$ function inside the ball $\{\mathbf{x}:|\mathbf{x}| \leq a\}$, where $k$ is defined below. Denote the Fourier transform and its spherical partial sum by

$$
\hat{f}(\mu):=\frac{1}{(2 \pi)^{n}} \int_{\mathbf{R}^{n}} f(\mathbf{x}) e^{-i\langle\mu, \mathbf{x}\rangle} d \mathbf{x}, \quad f_{R}(\mathbf{x}):=\int_{|\mu| \leq R} \hat{f}(\mu) e^{i\langle\mu, \mathbf{x}\rangle} d \mu .
$$

(i) If $n=1,2$ then $\lim _{R \uparrow \infty} f_{R}(0)=f(0)$.

(ii) If $n \geq 3$, then $\lim _{R \uparrow \infty} f_{R}(0)=f(0)$ if and only if

$$
\int_{|\mathbf{x}|=a} f d \sigma=0, \ldots, \int_{|\mathbf{x}|=a} \frac{\partial^{k} f}{\partial r^{k}} d \sigma=0,
$$

where $k=k(n):=\left[\frac{1}{2}(n-3)\right]$ and $d \sigma$ is the surface measure on the sphere of radius $a$. If $j(f):=\min \left\{j: 0 \leq j \leq k, \int_{|x|=a}\left(\partial^{j} f / \partial r^{j}\right) d \sigma \neq 0\right\}$, then limsup $\sup _{R \uparrow \infty} R^{-\nu}\left[f_{R}(0)-f(0)\right]>0, \liminf _{R \uparrow \infty} R^{-\nu}\left[f_{R}(0)-f(0)\right]<0$, where $\nu=\frac{1}{2}(n-3)-j(f) \geq 0$.

(iii) If $f(\mathbf{x})=F(|\mathbf{x}|)$ for some $F \in C^{2}[0, a]$, then the Fourier inversion takes place for any $\mathbf{x} \neq 0$ and $n \geq 1$ :

$$
\lim _{R \uparrow \infty} f_{R}(\mathbf{x})= \begin{cases}f(\mathbf{x}) & \text { if } 0<|\mathbf{x}| \neq a \\ \frac{1}{2} f(\mathbf{x}) & |\mathbf{x}|=a\end{cases}
$$

without any supplementary conditions.

The above result allows one to treat the pointwise invertibility of Fourier transforms without requiring that the Fourier transform be integrable. The

Received by the editors November 25, 1991.

1991 Mathematics Subject Classification. Primary 42B10; Secondary 33C10. 
conditions are sharp, in the sense that if any one of the surface integrals is nonzero, then the spherical partial sum diverges when $R \uparrow \infty$ at the indicated rate.

\section{Notation AND DEFinitions}

The volume of the $n$-dimensional unit ball in $\mathbf{R}^{n}$ is denoted $v_{n}=$ $\pi^{n / 2} / \Gamma(1+n / 2)$ while the $(n-1)$-dimensional measure of the unit sphere is $\omega_{n-1}=n v_{n}$. These may be computed in spherical coordinates as

$$
\begin{aligned}
v_{n} & =\omega_{n-2} \int_{0}^{1} \int_{0}^{\pi} r^{n-1} \sin ^{n-2} \theta d r d \theta \\
& =\frac{\omega_{n-2}}{n} \int_{0}^{\pi} \sin ^{n-2} \theta d \theta
\end{aligned}
$$

Hence $\omega_{n-2} \int_{0}^{\pi} \sin ^{n-2} \theta d \theta=n v_{n}$. We note, for future reference, that $v_{n}=$ $(2 \pi / n) v_{n-2}, n \geq 3$.

The spherical Bessel function in $n$ dimensions [DM, p. 133] is defined by

$$
\begin{aligned}
& V_{1}(r)=\cos r \\
& V_{n}(r)=\frac{\int_{0}^{\pi} e^{i r \cos \theta} \sin ^{n-2} \theta d \theta}{\int_{0}^{\pi} \sin ^{n-2} \theta d \theta}, \quad-\infty<r<\infty, n=2,3, \ldots
\end{aligned}
$$

Proposition 1.1. The spherical Bessel function satisfies

$$
\begin{gathered}
V_{n}(-r)=V_{n}(r) ; \\
\begin{array}{c}
(1.0) \\
(1.1)
\end{array} \\
V_{n}^{\prime \prime}+\frac{n-1}{r} V_{n}^{\prime}+V_{n}=0, \quad r \neq 0, \quad V_{n}(0)=1 ; \\
(1.3) \\
V_{n}(r)=1-\frac{r^{2}}{2 n}+\cdots, \quad r \rightarrow 0 ; \\
\text { (1.4) } V_{n}(r)=\left[\frac{2^{n / 2} \Gamma(n / 2)}{\left.\sqrt{2 \pi} r^{(n-1) / 2}\right]}\left[\cos \left(r-\frac{(n-1) \pi}{4}\right)+O\left(\frac{1}{r}\right)\right], \quad r \uparrow \infty .\right.
\end{gathered}
$$

Proof. The spherical Bessel functions are expressed in terms of the usual Bessel functions $J_{n}$ through the relation

$$
V_{n}(r)=2^{(n-2) / 2} \Gamma\left(\frac{n}{2}\right) \frac{J_{(n-2) / 2}(r)}{r^{(n-2) / 2}}, \quad r \neq 0 .
$$

The relations (1.0)-(1.4) then follow from the corresponding properties of $J_{n}$, proved in [W].

In what follows we shall occasionally identify a function on $[0, \infty)$ with its role as a radial function on $\mathbf{R}^{n}$. In particular, the independent variable can be interpreted either as a vector or a scalar argument. This identification will cause no confusion and will simplify the notation. 
The Dirichlet kernel in $n$ dimensions is

$$
\begin{aligned}
D_{R}^{n}(z) & :=\frac{1}{(2 \pi)^{n}} \int_{|\mu| \leq R} e^{i\langle\mu, z\rangle} d \mu \\
& =\frac{\omega_{n-2}}{(2 \pi)^{n}} \int_{0}^{R} \int_{0}^{\pi} e^{i \mu z \cos \theta} \sin ^{n-2} \theta d \theta \mu^{n-1} d \mu \\
& =\frac{n v_{n}}{(2 \pi)^{n}} \int_{0}^{R} V_{n}(\mu z) \mu^{n-1} d \mu .
\end{aligned}
$$

Proposition 1.2. The Dirichlet kernel has the following properties:

$$
\begin{gathered}
D_{R}^{n}(z)=\frac{2^{n / 2} \Gamma(n / 2) R^{(n-1) / 2}}{z^{(n+1) / 2}}\left[\sin \left(R z-(n-1) \frac{\pi}{4}\right)+O\left(\frac{1}{R}\right)\right], \\
z \neq 0, n=1,2, \ldots, R \uparrow \infty ; \\
D_{R}^{n}(z)=\frac{1}{2 \pi z} \frac{d}{d z} D_{R}^{n-2}(z), \quad z \neq 0, n=3,4, \ldots ; \\
D_{R}^{n}(-z)=D_{R}^{n}(z), \quad n=1,2, \ldots .
\end{gathered}
$$

Proof. Property (1.5) follows from the asymptotic property (1.4) of Bessel functions. In particular, for $n>1, D_{R}^{n}(z)$ is unbounded when $R \uparrow \infty$. Property (1.6) follows by applying the recurrence formula (1.2):

$$
\begin{aligned}
D_{R}^{n}(z) & =-\frac{n v_{n}}{(2 \pi)^{n}} \int_{0}^{R} \mu^{n-3} \frac{n-2}{z}\left(\frac{d}{d z}\right) V_{n-2}(\mu z) d \mu \\
& =\frac{-1}{2 \pi z} \frac{d}{d z} D_{R}^{(n-2)}(z) .
\end{aligned}
$$

Property (1.7) is immediate from the definition of $D_{R}^{n}(z)$.

\section{Proof of ConVergence/Divergence at the origin}

The spherical partial sum of the Fourier transform at $\mathbf{x}=0$ is

$$
\begin{aligned}
f_{R}(0) & =\int_{|\mu| \leq R} \hat{f}(\mu) d \mu=\frac{1}{(2 \pi)^{n}} \int_{|\mu| \leq R} \int_{|\mathbf{x}| \leq a} f(\mathbf{x}) e^{-i\langle\mu, \mathbf{x}\rangle} d \mu d \mathbf{x} \\
& =\int_{|\mathbf{x}| \leq a} f(\mathbf{x}) D_{R}^{(n)}(-\mathbf{x}) d x .
\end{aligned}
$$

In case $n=1, D_{R}^{1}(x)=(\sin R x) / \pi x$ and for any $C^{1}$ function $f$

$$
\lim _{R \uparrow \infty} \int_{-a}^{a} \frac{\sin R x}{\pi x} f(x) d x=f(0)
$$

from the one-dimensional theory of the Fourier integral.

In case $n=2, D_{R}^{2}(x)=R J_{1}(R x) / 2 \pi x$ and the spherical partial sum is

$$
\int_{|\mathbf{x}| \leq a} f(\mathbf{x}) D_{R}^{2}(\mathbf{x}) d \mathbf{x}=\int_{0}^{a} R J_{1}(R z)\left(M_{z} f\right) d z
$$

where $M_{z} f$ denotes the mean value on the sphere of radius $z$ centered at $(0,0)$. Without loss of generality, we may suppose that $f(0)=0$, since otherwise we may subtract that nonzero value and deal with the improper integral

$$
\int_{0}^{a} R J_{1}(R z) d z=J_{0}(0)-J_{0}(R a) \rightarrow 1, \quad R \uparrow \infty .
$$


We now integrate by parts, using $J_{0}^{\prime}=-J_{1}$, which reduces consideration to

$$
\int_{0}^{a} m(z) R J_{1}(R z) d z=J_{1}(a R) m(a)-\int_{0}^{a} J_{0}(a z) m^{\prime}(z) d z .
$$

The first term tends to zero by the asymptotics of $J_{0}$. To analyze the second, note that by the asymptotics of $J_{0}$, it tends to zero for any power $m^{\prime}(z)=z^{p}$, $p=1,2, \ldots$. But the continuous function $m^{\prime}$ can be uniformly approximated by a polynomial on the interval $[0, a]$ from which the result follows for all $f \in C^{1}$.

To study the convergence/divergence in higher dimensions, we use the properties of the Dirichlet kernel to write

$$
\begin{aligned}
\int_{|\mathbf{x}| \leq a} f(\mathbf{x}) D_{R}^{n}(\mathbf{x}) d \mathbf{x}= & \omega_{n-1} \int_{0}^{a}\left(M_{r} f\right) D_{R}^{n}(r) r^{n-1} d r \\
= & -\frac{\omega_{n-1}}{2 \pi} \int_{0}^{a}\left(M_{r} f\right) r^{n-2} \frac{d}{d r}\left[D_{R}^{n-2}(r)\right] d r \\
= & -\frac{\omega_{n-1}}{2 \pi}\left(M_{a} f\right) a^{n-2} D_{R}^{n-2}(a) \\
& +\frac{\omega_{n-1}}{2 \pi} \int_{0}^{a} D_{R}^{n-2}(r) \frac{d}{d r}\left[r^{n-2} M_{r} f\right] d r .
\end{aligned}
$$

Here we have used the notation $M_{r} f$ for the mean value of $f$ over the sphere of radius $a$ centered at $0 \in \mathbf{R}^{n}$. This formula reduces the convergence/divergence question to the behavior of the boundary term and that of a radial function in two lower dimensions, namely,

$$
r^{3-n} \frac{d}{d r}\left[r^{n-2} M_{r} f\right]=r \frac{d}{d r} M_{r} f+(n-2) M_{r} f
$$

If $n=3$ the second term tends to $f(0)$, by the one-dimensional theory of Fourier transforms. If, in addition, $M_{a} f=0$, then the entire sum converges to $f(0)$. Otherwise, when $M_{a} f \neq 0$, the first term is const $\times \sin R a$, which oscillates between nonzero limits when $R \uparrow \infty$.

Similarly if $n=4$ the second term tends to $f(0)$ by the two-dimensional case. If $M_{a} f=0$ then the spherical partial sum converges to $f(0)$. Otherwise the boundary term behaves as const $\times R^{1 / 2} \sin (a R-\pi / 4), R \uparrow \infty$, which proves the divergence in this case.

If $n>4$ we iterate the above procedure $N$ times to obtain

$$
\begin{aligned}
\int_{|\mathbf{x}| \leq a} f(\mathbf{x}) D_{R}^{n}(\mathbf{x}) d \mathbf{x}= & -\omega_{n-1} \sum_{k=1}^{N} \frac{D_{R}^{n-2 k}(a)}{(2 \pi)^{k}} f_{k}(a) \\
& +\frac{\omega_{n-1}}{(2 \pi)^{N}} \int_{0}^{a} D_{R}^{n-2 N}(r) \frac{d}{d r} f_{N}(r) d r,
\end{aligned}
$$

where the successive terms are defined by

$$
f_{0}(r)=r^{n-2} M_{r} f, f_{1}(r)=\frac{1}{r} \frac{d}{d r}\left(r^{n-2} M_{r} f\right), \ldots, f_{N}(r)=\left(\frac{1}{r} \frac{d}{d r}\right)^{N}\left(r^{n-2} M_{r} f\right) \text {. }
$$

If $n$ is odd, we perform this $N=(n-1) / 2$ times. The final integral converges to $f(0)$ by the one-dimensional theory. If all of the surface integrals are zero, then it follows that all of the terms in the above summation are zero and we have 
proved the required convergence. Otherwise, let $\nu$ be the first index for which $\int_{|\mathbf{x}|=a} \partial f^{j} / \partial r^{j} d \sigma \neq 0$. From the asymptotic form (1.5) of the Dirichet kernel we see that this sum consists of at most $k-\nu$ nonzero terms, the first of which is asymptotic to const $\times D_{R}^{n-2 \nu+2}(a)$, which behaves as $R^{(n-3-2 \nu) / 2}, R \uparrow \infty$. The remaining terms are estimated by $O\left(R^{j}\right)$, for $j<(n-3-2 \nu) / 2$. We have proved that $R^{-\nu} f_{R}(0)$ has the required fluctuation properties, in particular, the failure of convergence.

If $n$ is even, we perform this $N=(n-2) / 2$ times. The final integral converges to $f(0)$ by the two-dimensional theory. The remaining discussion is exactly as in the case of $n$ odd, as in the preceding paragraph. The proof is complete.

\section{PROOF OF CONVERGENCE FOR RADIAL FUNCTIONS}

Given $F(r), 0 \leq r \leq a$, we define the $n$-dimensional Fourier transform by

$$
\begin{aligned}
\widehat{F}_{n}(\mu) & =\frac{1}{(2 \pi)^{n}} \int_{|x| \leq a} F(|\mathbf{x}|) e^{-i\langle\mu, \mathbf{x}\rangle} d \mathbf{x} \\
& =\frac{1}{(2 \pi)^{n}} \int_{0}^{a} F(r) e^{-i \mu r \cos \theta} r^{n-1} \sin ^{n-2} \theta d r d \theta \\
& =\frac{n v_{n}}{(2 \pi)^{n}} \int_{0}^{a} F(r) r^{n-1} V_{n}(\mu r) d r .
\end{aligned}
$$

Lemma 3.1. The n-dimensional Fourier transform satisfies

(i) $\widehat{F}_{n}(\mu)=O\left(1 / \mu^{(n+1) / 2}\right), \mu \rightarrow \infty$;

(ii) $\widehat{F}_{n}(\mu)=-\widehat{F}_{n-2}^{\prime}(\mu) / 2 \pi \mu, \mu \neq 0$.

Proof. From the recurrence formula (1.2) for the spherical Bessel function, we have

$$
\begin{aligned}
\widehat{F}_{n}(\mu) & =-\frac{n v_{n}}{(2 \pi)^{n}} \int_{0}^{a} F(r) r^{n-1} \frac{n-2}{r \mu^{2}} \frac{d}{d r} V_{n-2}(\mu r) d r \\
& =-\frac{n(n-2) v_{n}}{(2 \pi)^{n} \mu^{2}}\left(F(a) a^{n-2} V_{n-2}(a \mu)+\int_{0}^{a} \frac{d}{d r}\left[r^{n-3} F(r)\right] V_{n-2}(\mu r)\right) .
\end{aligned}
$$

From the asymptotic behavior (1.4) of the spherical Bessel function, $V_{n}(\mu a)$ $=O\left(1 / \mu^{(n-1) / 2}\right)$. The final integral can be integrated-by-parts once again and the first result follows.

To prove the second, write

$$
\begin{aligned}
\widehat{F}_{n}(\mu) & =\frac{-n v_{n}(n-2)}{(2 \pi)^{n} \mu} \frac{d}{d \mu} \int_{0}^{a} F(r) r^{n-3} V_{n-2}(r \mu) d r \\
& =-\frac{1}{2 \pi \mu} \widehat{F}_{n-2}^{\prime}(\mu) . \quad
\end{aligned}
$$


With this established, we pass to the $n$-dimensional spherical partial sum

$$
\begin{aligned}
n v_{n} & \int_{0}^{R} \widehat{F}_{n}(\mu) \mu^{n-1} V_{n}(r \mu) d \mu \\
& =\frac{-n v_{n}}{2 \pi} \int_{0}^{R} \widehat{F}_{n-2}^{\prime}(\mu) \mu^{n-2} V_{n}(r \mu) d \mu \\
& =\frac{n v_{n}}{2 \pi}\left[\widehat{F}_{n}(R) R^{n-2} V_{n}(r R)-\int_{0}^{R} \widehat{F}_{n-2}(\mu) \frac{d}{d \mu}\left(\mu^{n-2} V_{n}(r \mu)\right) d \mu\right] .
\end{aligned}
$$

When $R \uparrow \infty$, the first term tends to zero for $r \neq 0$ by the above estimates. The second term for $r \neq 0$ can be written using the recurrence formula (1.2) as

$$
\frac{n v_{n}}{2 \pi} \int_{0}^{R} \widehat{F}_{n-2}(\mu) \frac{d}{d \mu}\left(\mu^{n-2}-\frac{-(n-2)}{r^{2} \mu} \frac{d}{d \mu} V_{n-2}(r \mu)\right) d \mu .
$$

The Bessel differential equation (1.1), written in the form

$$
\frac{d}{d \mu}\left(\mu^{n-3} \frac{d}{d \mu} V_{n-2}(r \mu)\right)+r^{2} \mu^{n-3} V_{n-2}(r \mu)=0,
$$

is then used to simplify the integrand to obtain

$$
\frac{n(n-2) v_{n}}{2 \pi} \int_{0}^{R} \widehat{F}_{n-2}(\mu) \mu^{n-3} V_{n-2}(r \mu) d \mu .
$$

We have proved that for $r \neq 0$ and $n=3,4, \ldots$

$$
\begin{aligned}
\lim _{R \uparrow \infty} & n v_{n} \int_{0}^{R} \widehat{F}_{n}(\mu) \mu^{n-1} V_{n}(r \mu) d \mu \\
& =\lim _{R \uparrow \infty}(n-2) v_{n-2} \int_{0}^{R} \widehat{F}_{n-2}(\mu) \mu^{n-3} V_{n-2}(r \mu) d \mu .
\end{aligned}
$$

This allows us to pass from convergence in dimension $n-2$ to convergence in dimension $n$. In case of one dimension, convergence follows from the onedimensional theory of the Fourier transform:

$$
\begin{aligned}
\lim _{R \uparrow \infty} v_{1} \int_{0}^{R} V_{1}(r \mu) \widehat{F}_{1}(\mu) d \mu & =\lim _{R \uparrow \infty} 2 \int_{0}^{R} \cos \mu r \widehat{F}_{1}(\mu) d \mu \\
& =\frac{1}{2}[f(r+0)+f(r-0)], \quad 0 \leq r \leq \infty .
\end{aligned}
$$

Applying the above argument gives convergence in dimensions $3,5,7, \ldots$ In case of two dimensions, convergence follows from the theory of the Hankel transform (for example, [S, p. 52])

$$
\begin{aligned}
\lim _{R \uparrow \infty} 2 v_{2} \int_{0}^{R} V_{2}(\mu r) \mu \widehat{F}_{2}(\mu) d \mu & =\lim _{R \uparrow \infty} 2 \pi \int_{0}^{R} J_{0}(\mu r) \mu \widehat{F}_{2}(\mu) d \mu \\
& =\frac{1}{2}[f(r+0)+f(r-0)], \quad 0<r<\infty .
\end{aligned}
$$

The above reduction now allows us to conclude convergence in dimensions $4,6,8, \ldots$. 


\section{EXAMPLES AND OBSERVATIONS}

1. The simplest example of the above divergence phenomenon is for the indicator function of the ball in $\mathbf{R}^{3}$. The Fourier transform is

$$
\begin{aligned}
\hat{f}(\mu) & =\frac{1}{(2 \pi)^{3}} \int_{|x| \leq a} e^{-i\langle\mu, \mathbf{x}\rangle} d \mathbf{x}=\frac{1}{2 \pi^{2} \mu} \int_{0}^{a} \sin r \mu r d r \\
& =\frac{1}{2 \pi^{2}}\left(\frac{a \cos a \mu}{\mu^{2}}-\frac{\sin a \mu}{\mu^{3}}\right), \quad \mu \neq 0 .
\end{aligned}
$$

The spherical partial sum is

$$
f_{R}(0)=4 \pi \int_{0}^{R} \hat{f}(\mu) \mu^{2} d \mu=-\frac{2}{\pi} \int_{0}^{R}\left[a \cos a \mu-\frac{\sin a \mu}{\mu}\right] d \mu .
$$

The second term is a convergent improper integral and the first integral is explicitly evaluated. Thus we have

$$
f_{R}(0)=1-\frac{2}{\pi} \sin R a+o(1), \quad R \uparrow \infty .
$$

Therefore we see explicitly that

$$
\limsup _{R \uparrow \infty} f_{R}(0)=1+\frac{2}{\pi}, \quad \liminf _{R \uparrow \infty} f_{R}(0)=1-\frac{2}{\pi} .
$$

This is reminiscent of the Gibbs phenomenon for one-dimensional Fourier series, where the graphs of the partial sums fill out an entire interval. In the present situation we have the additional fact that the partial sums diverge at a point. This is impossible for the one-dimensional Fourier series of a piecewise smooth function.

2. The above example can be modified to demonstrate the failure of localization in higher-dimensional Fourier analysis. Let the indicator function of the annulus be defined by $f(\mathbf{x})=1$ for $a<|\mathbf{x}|<b$, and $f(\mathbf{x})=0$ elsewhere. Clearly $f(\mathbf{x})=0$ in the ball $|\mathbf{x}|<a$. But from the computations in the above example, we have $\liminf \operatorname{in}_{R \uparrow \infty} f_{R}(0)<0<\lim \sup _{R \uparrow \infty} f_{R}(0)$; the spherical partial sum at the origin oscillates indefinitely although the function is zero in a neighborhood of the origin.

3. By going to higher dimensions we can produce a function that is zero in a neighborhood of the origin and whose spherical partial sum is unbounded. Indeed, following part (ii) of the main theorem, we can choose $f$ so that the unboundedness will be of the order $R^{(n-3) / 2}, R \uparrow \infty$ in dimension $n$.

\section{ACKNOWLDEGMENT}

We would like to thank George Gasper, Elton P. Hsu, and S. R. S. Varadhan for helpful conversations

\section{REFERENCES}

[DM] H. Dym and H. P. McKean, Fourier series and integrals, Academic Press, New York, 1972.

[S] I. Sneddon, Fourier transforms, McGraw Hill, New York, 1951.

[W] G. N. Watson, A treatise on the theory of Bessel functions, 2nd ed., Cambridge Univ. Press, London and New York, 1944. 
[GP] A. Gray and M. Pinsky, Gibbs' phenomenon for Fourier-Bessel series, Expositiones Math. (1992) (to appear).

[PST] M. Pinsky, N. Stanton, and P. Trapa, Fourier series of radial functions in several variables, J. Funct. Anal. (1993) (to appear).

Department of Mathematics, Northwestern University, Evanston, Illinols 602082730

E-mail address: m_pinsky@math.nwu.edu 